\title{
Domain-wall dynamics at micropatterned constrictions in ferromagnetic (Ga, Mn)As epilayers
}

\author{
J. Honolka, ${ }^{\text {a) }}$ S. Masmanidis, H. X. Tang, and M. L. Roukes \\ Condensed Matter Physics 114-36, California Institute of Technology, Pasadena, California 91125 \\ D. D. Awschalom \\ Department of Physics, University of California, Santa Barbara, California 93106
}

(Received 2 August 2004; accepted 19 December 2004; published online 4 March 2005)

\begin{abstract}
The influence of sub- $\mu \mathrm{m}$ geometric constrictions on $90^{\circ}$ magnetic domain-wall nucleation and propagation in stripes of ferromagnetic $\left(\mathrm{Ga}_{0.95}, \mathrm{Mn}_{0.05}\right)$ As was explored. Measurements of the magnetic switching behavior were performed during ramping of an external magnetic field at constant rate and at constant field in the time domain. Demagnetizing fields are found to play a crucial role in the switching behavior around the region of the constriction. Depending on the sample's initial magnetization the constriction can either assist domain-wall nucleation or hinder its propagation. @ 2005 American Institute of Physics. [DOI: 10.1063/1.1861512]
\end{abstract}

\section{INTRODUCTION}

The ability to make standard semiconductors ferromagnetic by heavy doping with magnetic impurities has renewed the general interest in the field of spintronics and has triggered ideas to use the spin degrees of freedom of carriers and ions to build logical units in semiconductor devices. ${ }^{1}$ For one of the prominent materials, $(\mathrm{Ga}, \mathrm{Mn}) \mathrm{As}$, it was stated that for fundamental reasons the Curie temperature might not exceed values of $\sim 110 \mathrm{~K} .^{2}$ Recent studies, however, show that it is possible to push the Curie temperature in $(\mathrm{Ga}, \mathrm{Mn}) \mathrm{As}$ to higher values, ${ }^{3,4}$ nourishing the prospect of integrating these materials in room-temperature devices in the future. One interesting application is to use magnetic domain walls (DWs) in logic circuits, exploiting the fact that switching the magnetization direction leads to drastic changes in the resistance. For this to be realized it is of crucial importance to be able to control the dynamics of DWs in mesoscopic systems. External applied magnetic fields influence the dynamics but are usually nonlocal, which means they cannot address certain individual units and therefore can serve as a computational clock $^{5}$ or reset pulse on larger scales only. The focus of the work presented in this paper is on gaining local control over the dynamics of DWs by modulation of the shape anisotropy. We examined how a constriction of a micrometer scale affects the nucleation and propagation of single DWs along a bar-shaped channel made from a semiconducting ferromagnetic epilayer.

\section{SAMPLE FABRICATION AND PRINCIPLE OF MEASUREMENT}

A 100 -nm-thick $\left(\mathrm{Ga}_{0.95}, \mathrm{Mn}_{0.05}\right)$ As epilayer was patterned into 10- $\mu \mathrm{m}$-wide Hall bars oriented along the [100] direction. The original epilayers were grown by means of molecular-beam epitaxy at $250{ }^{\circ} \mathrm{C}$ on an insulating GaAs(001) substrate with a buffer layer. The epilayers exhibit a Curie temperature of $\sim 45 \mathrm{~K}$ and an in-plane magnetic

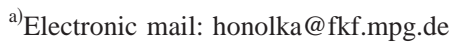

easy axis due to a compressive strain in the lattice. ${ }^{6}$ Three equidistant pairs of voltage probes, each of them about $1 \mu \mathrm{m}$ wide, were used for measuring longitudinal voltage drops across different sections of the Hall bar, denoted as $V_{L}, V_{M}$, and $V_{R}$ in Fig. 1. In some samples a constriction of $1-\mu \mathrm{m}$ width and $0.5-\mu \mathrm{m}$ length was added in the middle section of the bar, monitored by $V_{M}$. Two measurement schemes were employed: In one the signals were monitored via a standard lock-in technique while the external field was gradually ramped. In the other scheme the signals were fed to a multichannel oscilloscope to monitor the time evolution of the magnetization at constant magnetic field. All the experiments shown were carried out at a temperature of $4.2 \mathrm{~K}$.

Information on the state of magnetization in each of the sections can be extracted from the voltage signals $V_{i}$, where $i=L, M$, and $\mathrm{R}$, since the sheet resistance is related to the orientation of the magnetization by the anisotropic magnetoresistance (AMR) effect,

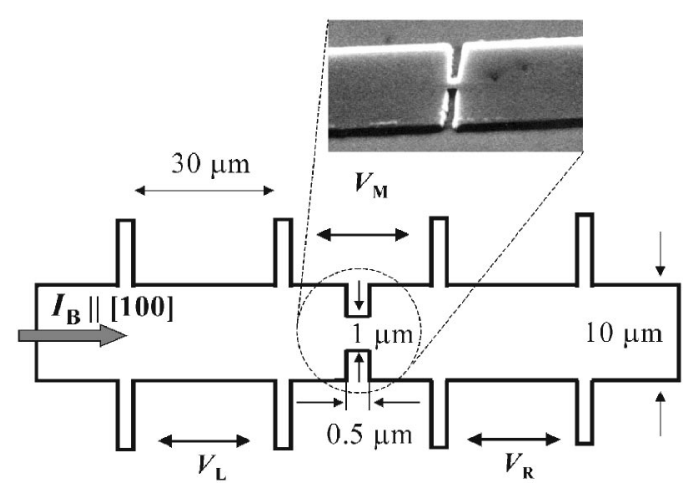

FIG. 1. Geometry of a bar-shaped sample with a $\left(1 \times 0.5 \mu \mathrm{m}^{2}\right)$ constriction in the middle section. Note that the constriction and the width of the voltage probes at the top and bottom are shown exaggerated with respect to the bar. A current $I_{B}$ of $10 \mu \mathrm{A}$ biases the sample and the voltages $V_{L}, V_{M}$, and $V_{R}$ along the three sections are picked up at the according probes. 


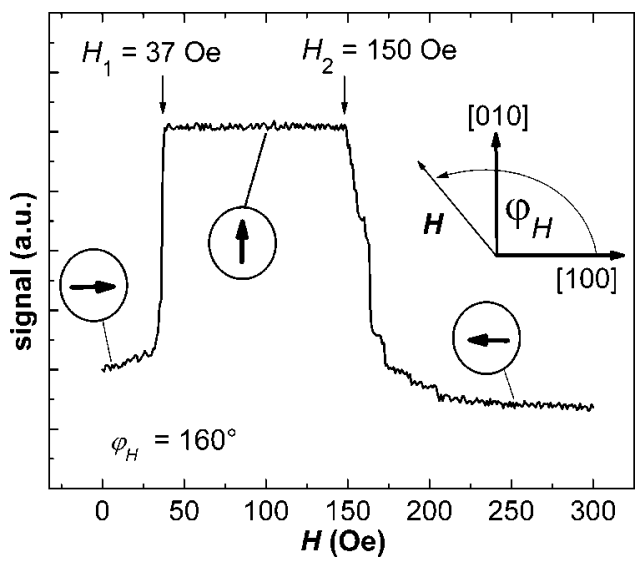

FIG. 2. Voltage across a $10-\mu \mathrm{m}$ bar without constriction during the constant ramping of the magnetic field at $\varphi_{H}=160^{\circ}$. Two jumps in $V_{M}$ at magnetic fields $H_{1}$ and $H_{2}$ indicate the sequential passing of two single $90^{\circ} \mathrm{DWs}$ while the field is gradually increased against the initial orientation of the magnetization (M initially along [100]).

$$
V_{i}=n_{i} I_{B}\left[\frac{\left(\rho_{11}-\rho_{\perp}\right)}{t} \cos ^{2} \varphi_{M}+\frac{\rho_{\perp}}{t}\right],
$$

where $I_{B}$ is the bias current and $\phi_{M}$ is the angle between the magnetization $\mathbf{M}$ and the current direction [100]. $\rho_{\|}\left(\rho_{\perp}\right)$ are the resistivities for currents parallel (perpendicular) to $\mathbf{M}$ normalized to the thickness $t$ of the film. $n_{i}$ denotes the effective number of serial sheet resistance squares in a section $i$. According to Fig. $1, V_{L}$ and $V_{R}$ each probe three (10 $\times 10 \mu \mathrm{m}^{2}$ ), whereas $\mathrm{V}_{M}$ probes approximately 3.5 squares since the constriction in the center contains two additional smaller $\left(0.5 \times 0.5 \mu \mathrm{m}^{2}\right)$ squares in parallel. Thus, for a given uniform reorientation of $\mathbf{M}$ the change in $V_{M}$ is expected to be the largest. In-plane magnetic fields of up to $5 \mathrm{kOe}$ were generated by a superconducting magnet. The angle $\varphi_{H}$ between the field orientation and the [100] direction within the plane of the film could be adjusted (accuracy $\sim \pm 5^{\circ}$ ) by rotating the sample relative to the magnet.

\section{EXPERIMENTAL RESULTS AND DISCUSSION}

The switching of the magnetization in uniform, nonconstricted bars of (Ga, Mn)As triggered by an applied magnetic field is known to be propagation dominated, ${ }^{7}$ that is, once a DW is nucleated it propagates rapidly along the sample. Hence, the samples switch their magnetization state in a single DW process. From time-domain measurements we know that nucleation in our samples always occurs at one of the ends of the Hall bar, possibly within the area of the big contact pads for the bias-current connection.

The switching behavior of a sample without a constriction is shown in Fig. 2, where the magnetic field is ramped in a direction of $20^{\circ}$ away from $[-100]$, that is $\varphi_{H}=160^{\circ}$. The initial magnetization state was along [100]. Two switching events are seen at magnetic fields of $H_{1}$ and $H_{2}$, indicating the sequential passage of two single $90^{\circ} \mathrm{DWs}$ while the field is gradually increased against the initial orientation of the magnetization. M, initially oriented along [100], switches to $[-100]$ via an intermediate orientation [010] reflecting the cubic magnetocrystalline anisotropy of the material. The ori- entation of the Hall bar is chosen along the [100] magnetic easy axis direction to maximize the change in voltage during magnetic transitions. According to (1), the magnitude of the jumps for the two transitions are given by $\Delta V_{i}=\mp n_{i} I_{B}\left(\rho_{\|}\right.$ $\left.-\rho_{\perp}\right) / t$. The switching fields $H_{1}$ and $H_{2}$ both depend on the angle $\varphi_{H}$. For more details on this issue we refer to a previous publication. ${ }^{6}$

From the switching fields we can estimate the nucleation energy of a DW per volume and magnetization assuming the relation $\varepsilon_{90^{\circ}} \cong\left(\mathbf{M}_{f}-\mathbf{M}_{i}\right) \mathbf{H}_{c}$. Here $\mathbf{M}_{f}$ and $\mathbf{M}_{i}$ stand for the initial and final magnetization states of the transition. We get the expression

$$
\varepsilon_{90^{\circ}} / M \cong \sqrt{2} \mathbf{H}_{c} \cos \left(\varphi_{H} \mp 135^{\circ}\right)
$$

for the case of the transitions $[100] \rightarrow[010]$ and $[010]$ $\rightarrow[-100]$ using the minus and plus signs, respectively. Plugging in the measured switching fields of $H_{1}=37$ Oe and $H_{2}$ $=150$ Oe shown in Fig. 2 with $\varphi_{H}=(160 \pm 5)^{\circ}$, the DW energy per volume and magnetization is $(47.4 \pm 6)$ and $(89.7 \pm 20)$ Oe for the two transitions, respectively.

The introduction of a submicron constriction changes the dynamics of the DW processes compared to the uniform Hall bars. For our samples with a Mn concentration of about 5\% we measured a magnetization of about $M=21 \mathrm{emu} / \mathrm{cm}^{3}$, and, thus, we expect local demagnetizing fields in the constriction region of the order of $4 \pi 0.055 M=14$ Oe. Here we modeled the constriction region as a flat ellipsoid with semiaxes $a>b>c$, where $c$ is the epilayer thickness. With $b / a$ $=0.5$ and $c / a=0.1$ the demagnetizing factor of $N / 4 \pi$ $=0.055$ for a uniform magnetization along the $a$ axis or [010] orientation. ${ }^{9}$ The contribution of demagnetizing fields to the magnetostatic energy of the system is comparable to that from the applied external fields, so significant changes in the DW behavior are expected near the constriction region. Figure 3 shows the voltages $V_{L}, V_{M}$, and $V_{R}$ while the magnetic field is ramped from zero field to 220 Oe at a speed of $2 \mathrm{Oe} / \mathrm{s}$ and an angle of $\varphi_{H}=150^{\circ}$. Prior to the ramping the sample was saturated at $H_{s}=5 \mathrm{kOe}$ along [100]. $V_{L}$ and $V_{R}$ switch in a similar manner, as for the case without a constriction, showing two transitions; however, the signal $V_{M}$ across the constriction shows two additional steps, indicating that the switching process now involves more than one DW.

We interpret the results of our measurements as follows: Two DWs propagate from both ends of the sample towards the constriction at $H_{1} \sim 38$ Oe. $\left(V_{L}\right.$ seems to switch a little earlier indicating that the nucleation energy is slightly lower at the reservoir on the left side.) While both $V_{L}$ and $V_{R}$ are fully switched at around $45 \mathrm{Oe}, V_{M}$ enters a plateau state and is not completely reversed until the field is further increased to 53 Oe. The two-step switching process at the constriction is a result of the strong local demagnetizing field. The orientation of $\mathbf{M}$ along [100] is favored since, in the case of an orientation along [010], $\mathbf{M}$ opposes the demagnetizing field. In the constriction region the magnetization therefore tends to remain in the [100] orientation. The transition from [100] to [010] is finally completed at increased fields when the Zeeman energy $M H \cos \left(\varphi_{H}-\varphi_{M}\right)$ overcomes the energy contribution of the demagnetizing field. The measured increasement is $15 \mathrm{Oe}$, which fits well to the demagnetizing 


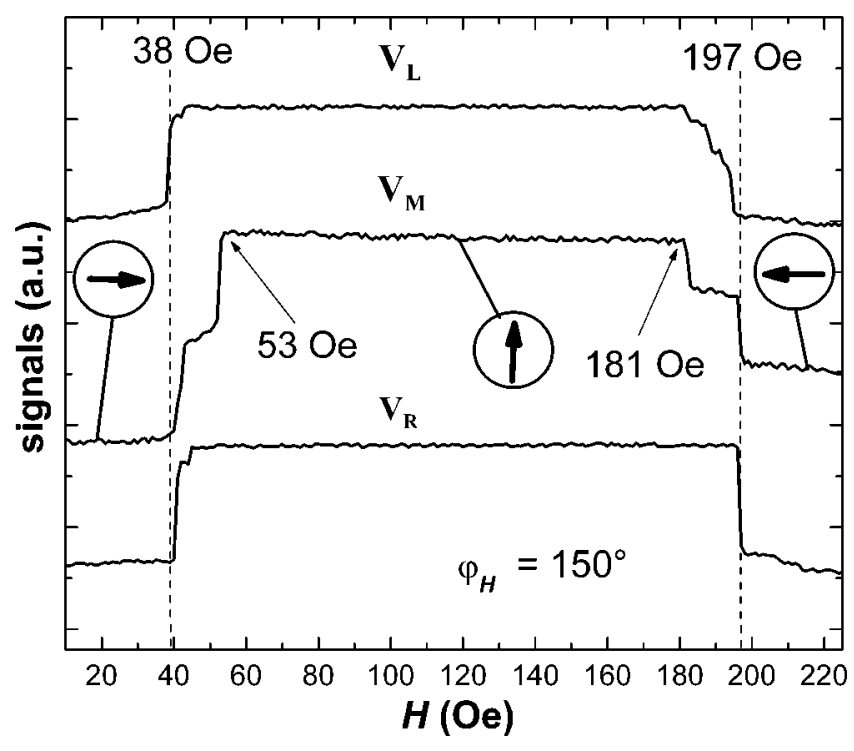

FIG. 3. Voltages $V_{L}, V_{M}$, and $V_{R}$ with constriction during the constant ramping of the magnetic field against the initial orientation of the magnetization (M initially along [100]) at $\varphi_{H}=150^{\circ}$. While two jumps are seen in $V_{L}$ and $V_{R}$ two additional plateaus appear in the voltage $V_{M}$ across the section containing the constriction. The orientation of $\mathbf{M}$ in the three single domain states are represented by the arrows.

field of 14 Oe predicted by our model. Thus, in the region of the plateau seen the magnetic configuration should look like an "island," which blocks passage to the DWs. The configuration is illustrated schematically in Fig. 4(a), however we want to point out that the exact shape cannot be derived from our data.

Conversely for the case of the second transition from $[010]$ to $[-100]$, the demagnetizing field has the effect of destablizing the unfavorable [010] orientation of $\mathbf{M}$. The center section switches first nucleating two DWs around the constriction forming a "bubble" around it [see Fig. 4(b)]. Further increase of the magnetic field increases the area of the bubble seen as a slight slope in $V_{M}$ in Fig. 3. This can be observed even more clearly in the quasistatic measurements not presented here, which show that it is possible to blow up the bubble stepwise by gradually increasing the magnetic fields every $10 \mathrm{~min}$. We believe that the bubble increases its area by stretching outwards towards the sides of the Hall bar [see dotted lines in Fig. 4(b)] thereby increasing its energy, which scales with the surface area of the DW. At a critical field of $\sim 197 \mathrm{Oe}$, possibly when the DWs are fully stretched, they get depinned and propagate outwards along the Hall bar. It should be stressed here that the depinning happens at fields well below 245 Oe, the field at which we expect the nucleation of DWs from the far ends of the sample according to (2) with $\varphi_{H}=150^{\circ}$ and $\left(\varepsilon_{90^{\circ}} / M\right.$ $\cong 89.7 \mathrm{Oe}$ ).

To confirm our interpretation the process of the island and bubble formation was measured in the time domain at constant magnetic field. The field was chosen to be large enough to trigger the nucleation, but small enough to slow the DW down to obtain a sufficient time resolution. ${ }^{10}$ After saturating the sample with $5 \mathrm{kOe}$ along [100] the field was reversed to $79 \mathrm{Oe}$ at an angle of $\varphi_{H}=70^{\circ}$ which was followed by the domain-wall nucleation approximately $30 \mathrm{~s}$

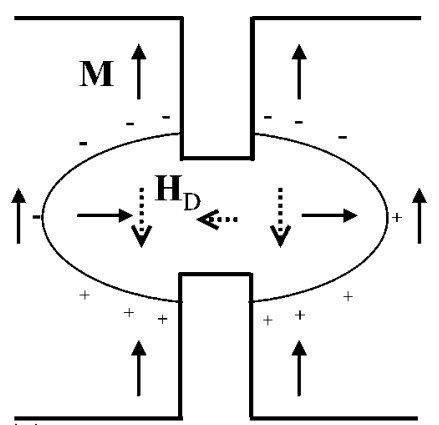

(a)

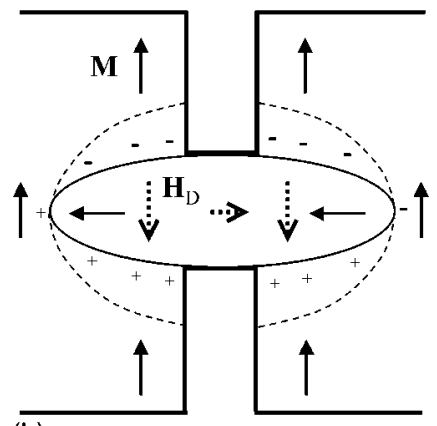

(b)

FIG. 4. Schematic model of the DW configurations in the constriction region for the island and bubble cases. DWs are drawn in full black lines, $\mathbf{M}$ in full arrows, and the demagnetizing field $\mathbf{H}_{D}$ in dotted arrows. (a) Island configuration: Within the constriction $\mathbf{M}$ points to the right, which corresponds to the first plateau at lower fields seen in Fig. 3. (b) Bubble configuration: Within the constriction $\mathbf{M}$ points to the left, which corresponds to the second plateau at higher fields seen in Fig. 3. At increasing magnetic fields the bubble increases its area by stretching outwards towards the sides of the Hall bar (see dotted lines) thereby increasing its energy, which scales with the surface area of the DW.

later. Note that at this angle $\varphi_{H}$ according to (2) with $\left(\varepsilon_{90^{\circ}} / M \cong 47.4\right.$ Oe $)$ we indeed expect the first DW to be nucleated at around $(79 \pm 10)$ Oe. Figure 5 shows the result of this experiment, which proves the formation of an island. $V_{R}$ switches about $9 \mathrm{~s}$ earlier than $V_{L}$ while $V_{M}$ switches twice. The total height of the jump in $V_{M}$ is slightly smaller than that seen for $V_{L}$ and $V_{R}$, indicating that the central region has not fully switched its magnetization.

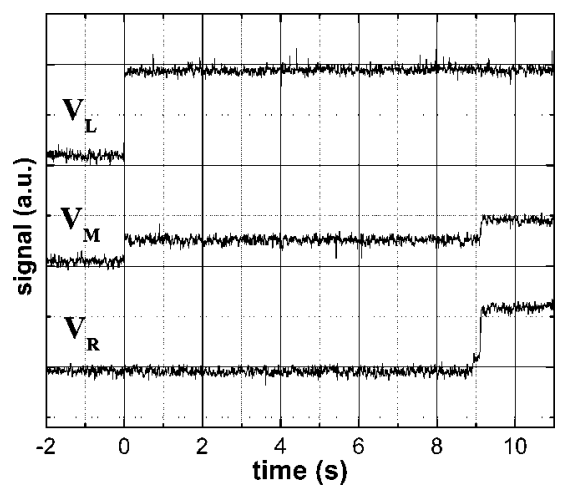

FIG. 5. Voltages $V_{L}, V_{M}$, and $V_{R}$ in the time domain at constant magnetic field. The field is applied against the initial magnetization ( $\mathbf{M}$ initially along [100]) with $79 \mathrm{Oe}$ and $\varphi_{H}=70^{\circ} . V_{R}$ switches about $9 \mathrm{~s}$ after $V_{L}$ proving the two-DW process. $V_{M}$ switches in two steps however the total height indicates that the middle section containing the constriction has not switched entirely. 


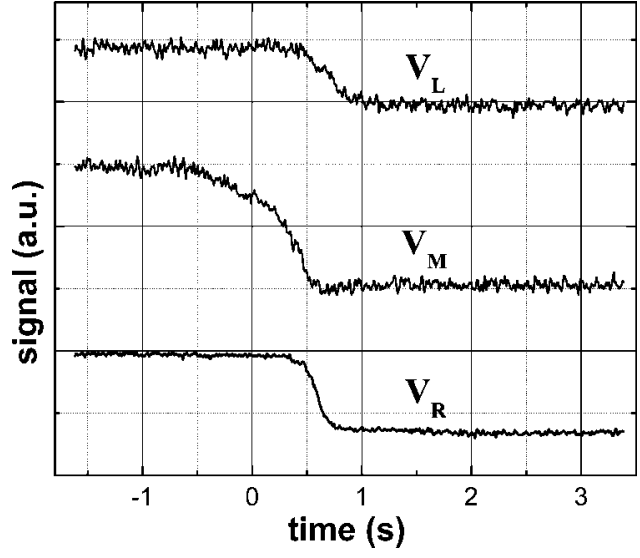

FIG. 6. Voltages $V_{L}, V_{M}$, and $V_{R}$ in the time domain at constant magnetic field. The sample was initially prepared in the [010] single domain state. The constant field of 67 Oe was applied along $[-100]\left(\varphi_{H}=180^{\circ}\right) . V_{M}$ switches before $V_{L}$ and $V_{R}$ indicating the nucleation of a pair of DWs at the constriction.

In order to look at the bubble formation the system was prepared in the [010] single domain state. A magnetic field of $5 \mathrm{kOe}$ was applied along [010] and slowly ramped down to zero again. No switching was observed while reducing the field to zero. After that, the sample was rotated and a field of 67 Oe was applied along [-100]. From the result shown in Fig. 6 it is evident that the nucleation of DWs occurs at the constriction. $V_{M}$ is almost fully switched before changes in $V_{L}$ and $V_{R}$ are observed. In contrast with the case of the island formation, here the total change in $V_{M}$ is larger than $V_{L}$ and $V_{R}$, suggesting the applied field was sufficient to convert the entire central region into the final single domain state along [-100].

\section{CONCLUSION}

In conclusion we have shown that the demagnetizing fields induced at submicron-sized geometric constrictions in ferromagnetic semiconducting $(\mathrm{Ga}, \mathrm{Mn})$ As have a significant impact on the dynamics of domain-walls (DWs) nucleation and propagation. DWs are either nucleated at constrictions or hindered from passing them, depending on whether the initial magnetization state is parallel or perpendicular to the constriction. This effect is a joint consequence of first the cubic symmetry of the semiconductors magnetic anisotropy energy favoring the nucleation of $90^{\circ} \mathrm{DWs}$ and second the contribution of the demagnetizing fields to the magnetostatic energy favoring the orientation of the magnetization parallel to the constriction. The dynamics can be quantitatively described using a model that treats the constriction region as a flat ellipsoid.

From an applied point of view a constriction can thus be used as a unit that ejects DWs or blocks them. In the blocking case the functionality is switched off beyond a certain critical magnetic field allowing the DW to penetrate the constriction area whereas in the case of an ejection unit a critical field has to be applied to trigger the functionality. In a device comprising several of the discussed functional units, the modulation of the anisotropy energies by the local electric fields ${ }^{11}$ could give additional control over the triggering of certain units. Due to the significant changes of the resistance during the magnetic switching, the magnetic state of different sections in a logic unit can be read out using simple resistance measurement schemes similar to the ones used in this work.

\section{ACKNOWLEDGMENTS}

We are grateful for financial support from the DARPA/ SPINS Program and the Deutsche Forschungsgemeinschaft. For valuable discussions we would like to thank Professor P. E. Wigen.

${ }^{1}$ H. Ohno, JSAP 5, 4 (2002); S. A. Wolf et al., Science 294, 1488 (2001).

${ }^{2}$ K. W. Edmonds et al., Phys. Rev. Lett. 92, 037201 (2004).

${ }^{3}$ K. C. Ku et al., Appl. Phys. Lett. 82, 2302 (2003).

${ }^{4}$ Ahsan M. Nazmul, S. Sugahara, and M. Tanaka, Phys. Rev. B 67, 241308 (2003).

${ }^{5}$ D. A. Allwood et al., Science 296, 2003 (2002).

${ }^{6} \mathrm{H}$. Ohno, Science 281, 951 (1998).

${ }^{7}$ H. X. Tang, R. Kawakami, D. D. Awschalom, and M. L. Roukes, Phys. Rev. Lett. 90, 107201 (2003)

${ }^{8}$ S. J. Potashnik et al., Phys. Rev. B 66, 012408 (2002).

${ }^{9}$ J. A.Osborn, Phys. Rev. 67, 351 (1945).

${ }^{10}$ At fields close to the coercive field the propagation velocity of a DW should depend exponentially on the applied magnetic field since the process of movement is thermally activated. We saw that little changes of the constant magnetic field of the order of a few Gauss can alter the time scale of the dynamics from seconds to minutes.

${ }^{11}$ H. Ohno et al., Nature (London) 408, 944 (2000). 